\title{
SAT: A WEB-BASED INTERACTIVE ADVISOR FOR INVESTOR-READY BUSINESS PLANS
}

\author{
Dietmar Jannach \\ Institute of Applied Informatics, Klagenfurt University, Universitätsstraße 65, Klagenfurt, Austria \\ dietmar.jannach@uni-klu.ac.at \\ Uffe Bundgaard-Joergensen \\ InvestorNet \& Mermaid Venture, Scion-DTU, Diplomvej 381, Kongens Lyngby, Denmark \\ ubj@gate2growth.com
}

Keywords: Self-service applications, e-services, knowledge-based systems.

\begin{abstract}
The business plan is one of the essential tools for companies to attract investors and raise venture capital. Getting a business plan "investor ready" in that context means that it has to be professionally prepared such that it answers all questions of potential investors. This, however, requires in-depth knowledge of a typical investor's expectations and viewpoints, a type of knowledge which in particular first-time entrepreneurs or small companies do not dispose of. As a consequence, significant amounts of consulting hours are required to get the business plan ready to be presented to investors.

The goal of the SAT project presented in this paper is to provide an in-depth business plan advisory service over the Web. While current approaches in that area rely on static fill-out forms or checklists, the SAT tool is based on personalized interactive dialogs, knowledge-based input analysis and feedback generation, as well as on detailed financial calculations. Within the paper, we thus show how knowledge-based approaches can serve as a technological foundation for such next-generation electronic services and how the corresponding development and maintenance efforts can be minimized. The paper gives an overview on the general knowledge-based architecture of the system, discusses the integrated graphical modelling environment, and finally reports on experiences gained from the practical use of the tool.
\end{abstract}

\section{INTRODUCTION}

Getting a business plan "investor ready" is a challenging task in particular for young entrepreneurs or smaller companies with limited experience and background in acquiring investor capital. Although the importance of a business plan as one of the essential tools for attracting investors is widely known and lots of guides and further literature are available, our year-long experience shows that typical mistakes are still common. In the context of the commercialization of e-Business related innovations for instance, technological aspects are in many cases overly emphasized. At the same time, investors often complain that the investor's view is not properly addressed in the business plans they receive. Much too often aspects like experience of the management team, the internal return rate and exit scenarios for the investor, or detailed statements on financials and uncertainty factors are neglected. This has even lead to an EU sponsored training initiative called "Master Classes" where innovation professionals and entrepreneurs are introduced to the world of "investor thinking". Thus, these entrepreneurs or less-experienced companies often chose to utilise professional advice to increase their chances of raising capital for their business ideas. Professional counselling however requires highly-experienced advisors and hence further increases the amount of in-advance investments required for the planned commercialization.

The herein described SAT (self assessment tool) project was initiated with the goal to provide a new, high-quality advisory service on the Web. The resulting software solution should consequently be 
deployed in the context of an EU-funded Web portal for Business and Innovation Financing (Gate2Growth), which aims at bringing together entrepreneurs, innovation professionals and investors.

When examining the existing sources of information for entrepreneurs who search for innovation financing advice on the Web, one can observe that these sources mostly exist in the form of static fillout forms or checklists. In contrast to such static approaches, the SAT tool in its vision aims at providing a virtual advisor that simulates the behaviour of an experienced business consultant in different dimensions. This includes for instance that both the characteristics of the entrepreneur as well as the key aspects of the business idea are elicited in a highly personalized interactive dialog or interview. Technically, this means for instance that the sequence of questions can not be a static one and the system has to continuously react to the current user's inputs. In addition, such a system should also immediately react once it detects inconsistencies in the user's answers or identifies situations, in which additional hints or cross-references appear to be useful. For a general overview of these personalization opportunities in such e-service applications, see also (Jannach \& Kreutler, 2005).

As the provision of highly personalized applications is known to be a knowledge-intensive task (Kobsa et al. 2001), we have decided to follow a knowledgebased software development approach and base the SAT tool on the ADVISOR SUITE framework described in (Felfernig et al. 2007). The main advantages that we expected from the decision were as follows. First, the efforts for development and maintenance should be minimized as domain experts are given the opportunity to add and modify individual pieces of knowledge by themselves. In addition, by choosing a more general and domainindependent framework as a basis, future extensibility toward other types of online advisory services on the Web portal should be guaranteed.

Based on an analysis of the particular challenges and opportunities of a fully knowledge-based approach the paper shall thus demonstrate how - on the basis of a concrete example - these techniques can serve as a basis for next-generation intelligent electronic online services.

The rest of the paper is organized as follows. In the next section, we will sketch how the end user perceives the virtual advisor application and what aspects are covered during the business plan analysis phase. Next, we give an overview on technical aspects, in particular how the different required pieces of knowledge can be modelled within the graphical SAT knowledge acquisition environment. The paper ends with a discussion of first experiences both with respect to system development as well as end user feedback and a comparison to similar approaches.

\section{THE END USER'S VIEW}

Figure 1 depicts how the profile of the online user is interactively elicited. The system, which is optionally personificated by an animated avatar, guides the entrepreneur through a series of personalized questions. Throughout this systemdriven dialog, the user inputs are constantly monitored and the virtual advisor chooses the next

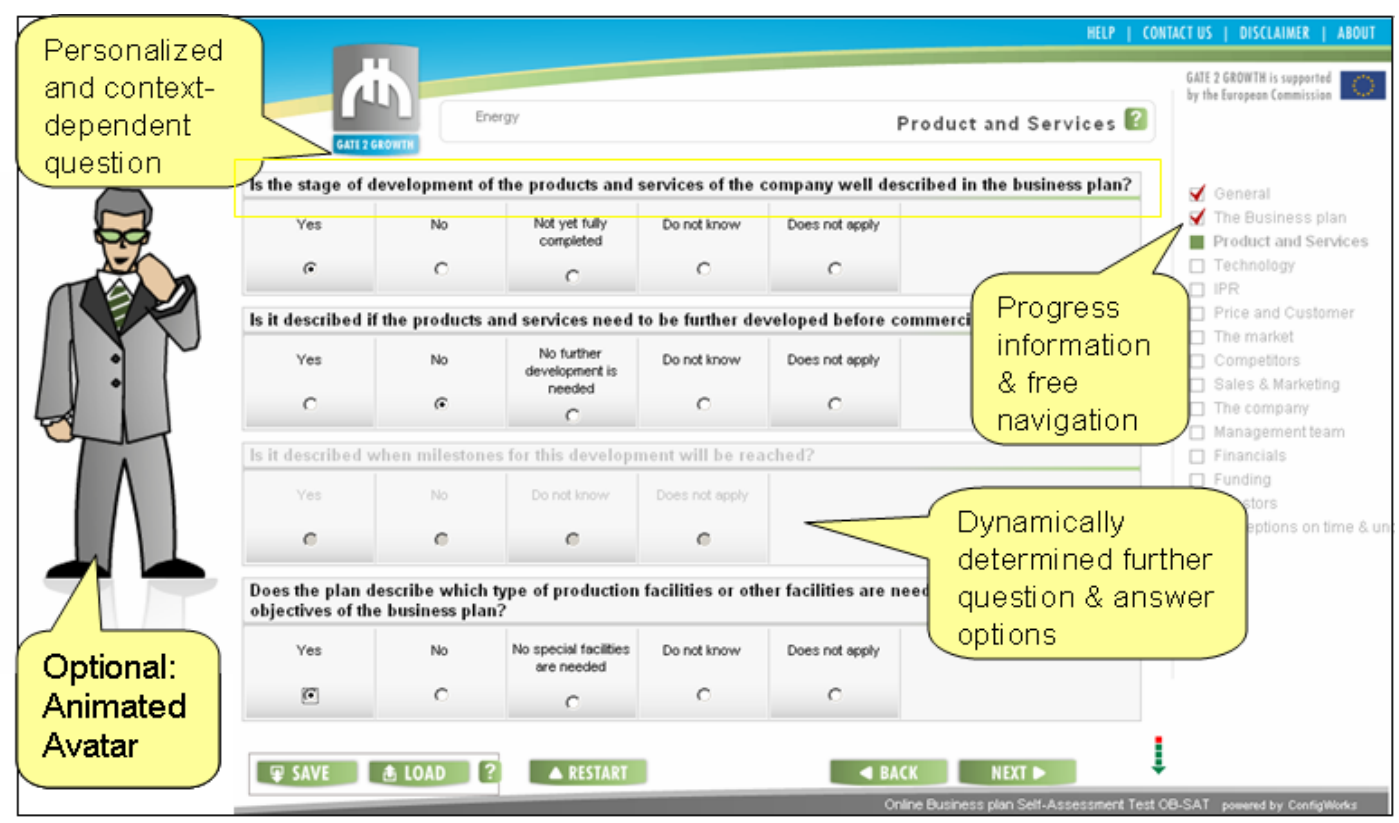

Figure 1: An interactive dialog page. 
questions to be asked based on the current state of the dialog. Depending on the current context, for instance, unnecessary questions may also be fully left out or specific textual variant might be asked when the user seeks for investors in a certain industry sector. When the system identifies (obviously) conflicting user statements, the question and answer dialog is interrupted and opportunistic hints and explanations are provided.

In order to give the user appropriate feedback about the progress, the dialog is structured into phases, see also the right hand side of the screen. Note that not all these phases are mandatory, i.e., when no detailed financial analysis is desired, individual parts of the dialog are automatically skipped (in contrast to more static approaches).

Once enough details have been provided, the system generates different types of feedback for the entrepreneur. The integrated analysis of the business plan given by the system has various facets (Figure 2). First, the completeness of the business plan content is evaluated based on a scoring mechanism along twelve different dimensions like management team, products and services, sales and marketing and so forth. The results of this scoring are visualized and summarized in terms of a "completeness radar" as shown in Figure 2. In the detailed feedback section, a comprehensive review of the user's statements and characteristics is given. For each of the twelve different subsections, about five to ten comments, explanations, or suggestions are proposed to the end user. Next, the "investor's view" is summarized, which is based on an in-depth analysis of various factors. The influence factors include the user's statements about financial aspects like expected profit and loss within the next years, an estimate of internal return rates, or fundingrelated issues like the percentage of ownership offered to the investor. The feedback given is split into a section in which key indicators for investors are calculated and a section which explains and comments how these indicators will be interpreted by the investors. The individual statements are then also condensed into compact aggregated advices (see also Figure 2).

Finally, the SAT system provides a detailed statement about the entrepreneur's attitude toward time preferences and uncertainty aspects: In this section, the given financial numbers are compared with the user's feedback on his estimate of future developments and preferred investment scenarios. One of the goals of this feedback section is thus to cross-check the plausibility of user responses.

\section{KNOWLEDGE-BASED SYSTEM DEVELOPMENT}

As can be seen from the short system description in the previous section, the application is knowledgeintensive, i.e., significant amounts of domain expertise have to be encoded in the background, from business plan analytics, over dialog management, to investor-specific financial

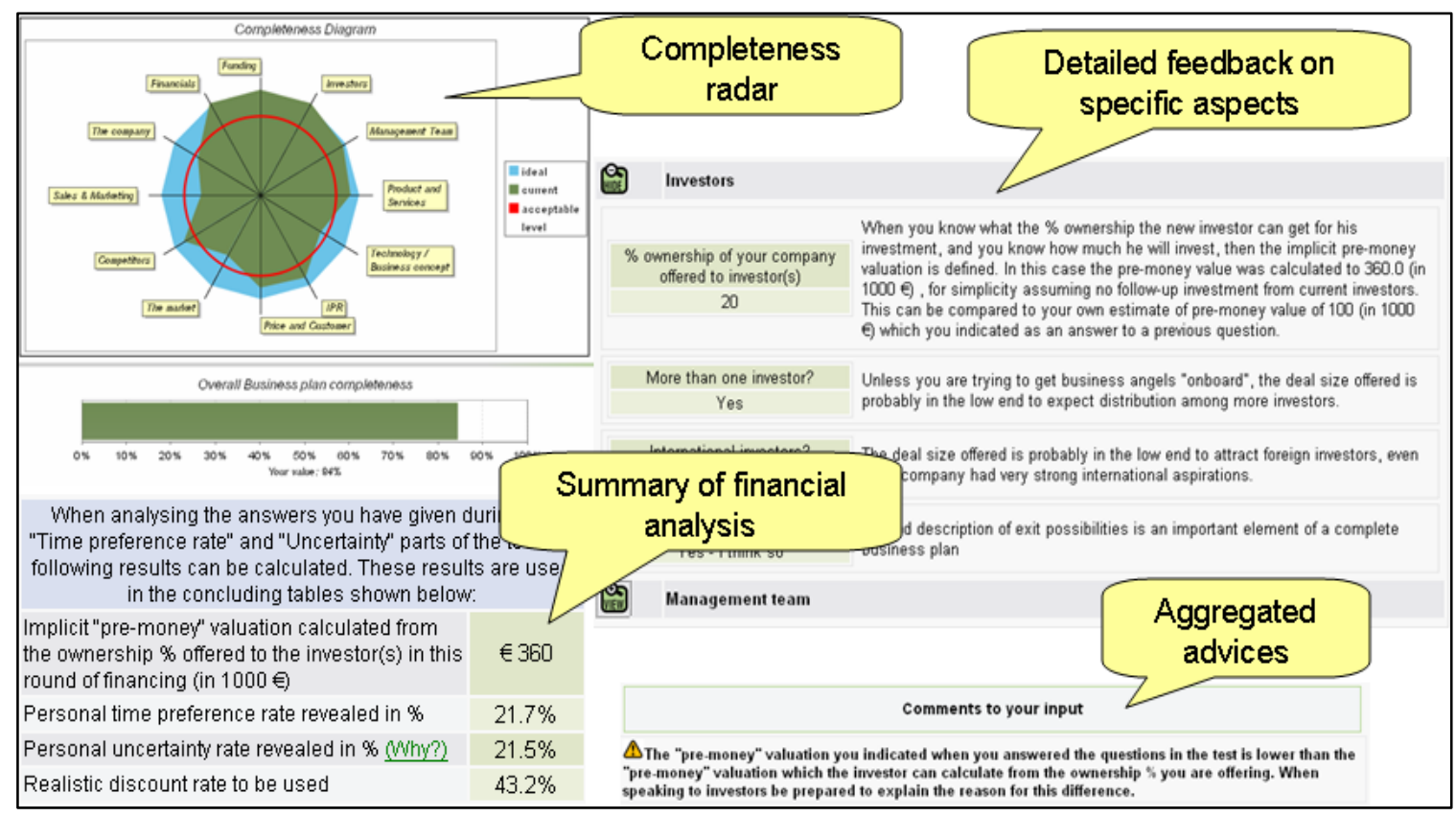

Figure 2: In-depth investor readiness analysis. 
calculation schemes. Consequently, aspects of knowledge acquisition and maintenance have to be in the centre of system design considerations: On the one hand the knowledge in the field constantly evolves while at the same time on the other hand the system should be continuously improved based on usage experiences or user feedback. In the subsequent sections, we will thus discuss how the different pieces of knowledge can be captured with the help of the SAT Modeling Environment and how knowledge acquisition and maintenance efforts can therefore be kept at a manageable level.

\subsection{The business plan profile}

The business plan and entrepreneur profile is the central element in the system's knowledge base and it is used to capture the key characteristics of the entrepreneur and the corresponding business plan to be analyzed. Technically, the profile consists of a set of variables $V$, each of them with a defined domain, which can be a finite enumeration of values or a scalar data type like integer or real. Each variable can also be set-valued, i.e., multiple values can be assigned to a variable at the same time. The actual values for a business plan analysis session are determined either by directly questioning the user (Figure 1) or by internally deriving the value based on defined business rules, for instance based on scoring functions.

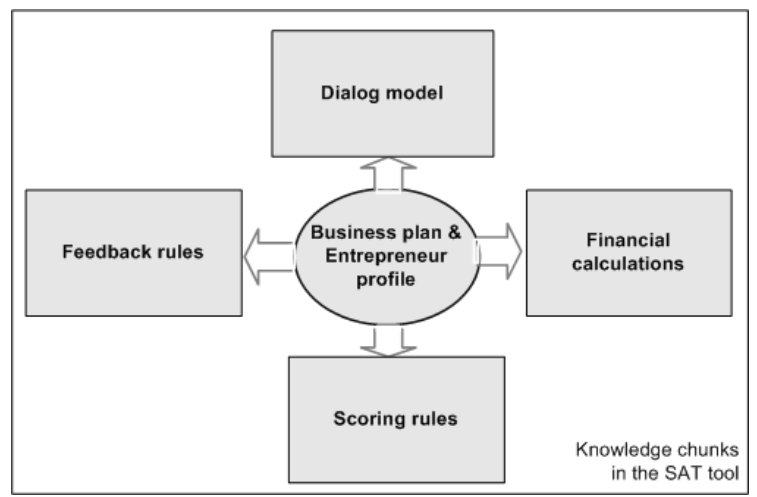

Figure 3: Knowledge chunks in the SAT tool.

Figure 3 sketches how the business plan profile $(B P)$ is related to the other pieces of knowledge in the SAT tool. When values for $B P$ should be directly acquired, the variables are referenced from the dialog model, in which the interactive elicitation process is defined.

The variables of $B P$ are of course also the starting point for defining the feedback generation process. First, they serve as an input to the internal derivation of additional values based on financial calculations or scoring rules. In addition, the variables of $B P$ are to be used in the definition of feedback rules that determine the set of analytical statements and hints to be displayed to the entrepreneur (see Figure 2).

\subsection{Feedback rules}

The knowledge base of feedback rules (KBFR) about the various aspects of the business plan to be analysed is the largest piece of domain expertise encoded in the system. Currently, the SAT tool comprises more than 450 of these statements.

In the SAT system, a feedback rule FR is represented as a tuple

$<$ ID, Group, Text, Order, Expression, Lang >.

Each feedback rule has a unique ID, belongs to a group, and has an associated multi-lingual, parameterizable textual statement as well as an optional order of display. The selection of appropriate feedback statements for a given business plan profile is determined by an expression over the variables of $B P$. An example for such a feedback rule that also includes derived variables from the financials module could be as follows.

"The discount rate of \$proposed_discount_rate\$ you have proposed to be used for the 'net present value' calculations is higher than the "time preference rate" you have revealed from answering other questions during the test (...)."

The corresponding rule could be for instance Display when

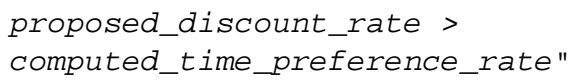

The symbols proposed_discount_rate and computed_time_preference_rate are variables from the Business plan Profile BP; the expression language used in the SAT tool comprises standard arithmetic and relational operators as well as logical operators like and, or, and not. The definition of feedback rules including the display conditions can be fully done with the help of graphical editors (see later sections). At run-time, the conditions are automatically evaluated by the system in the context of the current user session as to determine the subset of feedback texts to be displayed.

\subsection{The dialog model}

In contrast to the above-mentioned feedback rules, the definition of the dialog model describing for instance, which questions have to be displayed under what circumstances, is more complex.

In the SAT system, the possible flow of the systemuser dialog is modelled as a predicate-based finite state automaton (van Noord \& Gerdemann, 2001). The main advantage of this common dialog 
modelling approach is mainly that the resulting models can be easily understood and modified by using a corresponding graphical editor. Note that this aspect is of particular importance in the context of our work, in which we aim at enabling the domain expert himself to update the knowledge base as far as possible.

Within the described SAT system, the nodes and edges of graph can be annotated as follows (see Figure 4). As the nodes of the automaton correspond to dialog pages (Figure 1), we define the set of questions to be displayed on that page and also specify how each question should be rendered. Furthermore, we can make the display of a particular question (or answer option) dependent on previous user inputs in order to reach a more dynamic behaviour. The questions themselves again correspond to variables from the central Business plan Profile $(B P)$, i.e., when variables from $B P$ should be directly elicited by asking the user, these variables are annotated beforehand with appropriate text fragments, both for the question itself, the answer options, and optional explanations.

The second type of annotation is that for transition conditions which specify how the dialog shall proceed. Syntactically, these conditions are again expressions over variables of $B P$ which we consistently use in the modelling environment. Note that the system does not allow for indeterminism in the dialog graph. In order to support the user when modelling the dialog, the underlying ADVISOR SUITE system comprises a novel, built-in diagnosis mechanism (Felfernig et al., 2006) for finding inconsistencies or unreachable paths.

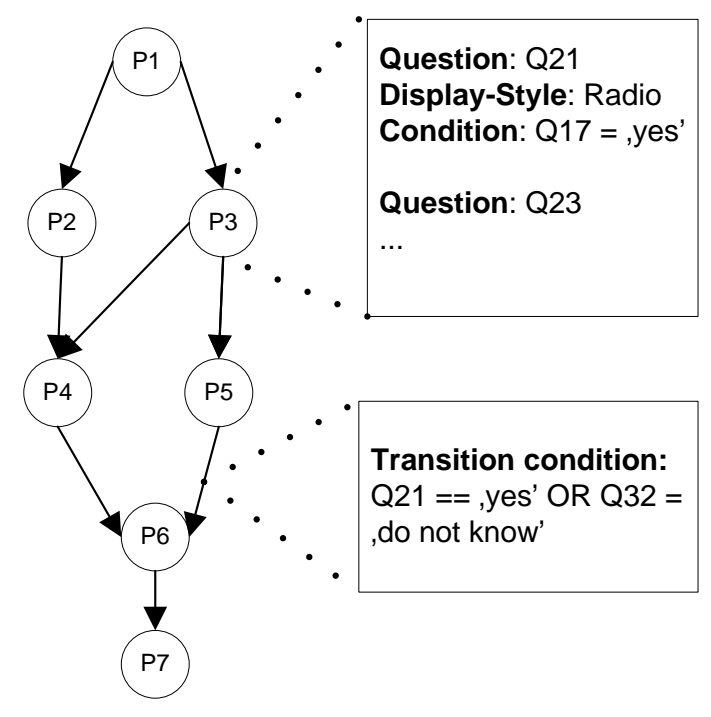

Figure 4: Annotated state diagram.
Also in the dialog model, the domain expert can specify when conditional hints should be displayed, if for instance the user has given inconsistent answers during the dialog. A detailed description of these features is omitted in this paper for sake of brevity. The development cycle of the SAT tool with regard to the dialog model is as follows. Once the dialog model has been completed or modified, a user interface generation task is initiated in which dynamic HTML pages (Java Server Pages in our case) are generated based on the definitions in the knowledge base. Thus, also the maintenance process for the personalized graphical user interface is supported in the modelling environment. Further details on this specific code generation process can be found in (Jannach \& Kreutler, 2004).

\subsection{Scoring schemes}

The evaluation of "investor readiness" of the business plan along different dimensions is based on a standard scoring mechanism and visualized by means of a completeness diagram (see Figure 2). Thus, for each of the twelve dimensions (like Marketing \& Sales, Technology and so forth) a scoring function has to be defined. In the SAT modelling tool, each dimension is therefore associated with a set of variables from the business plan profile $B P$ and for each possible value of a variable a numerical and normalized score from 0 to 10 is defined. The definition of this score value can be either done by assigning concrete numbers for enumerated domains as well as by means of a mapping function for variables with continuous domains.

At run time, the actual values in the $B P$ are evaluated and an overall cumulative scoring value per dimension is determined based on a MultiAttribute-Utility-Theory calculation (von Winterfeld et al, 1986). Note that the scoring dimensions themselves are again treated as variables in $B P$, which means that one can also define expressions in personalization or feedback rules that take the current value of the scoring result into account.

\subsection{Financial calculations}

An integrated evaluation of a business plan with respect to attractiveness to potential investors requires an in-depth analysis of financial aspects and accompanying uncertainty factors. Such calculations are based on various inputs such as estimated profit and loss figures for the upcoming years, a projection of these numbers for subsequent years, or time points and amounts of future investments. From these and other inputs, the "investor's view", i.e., an 

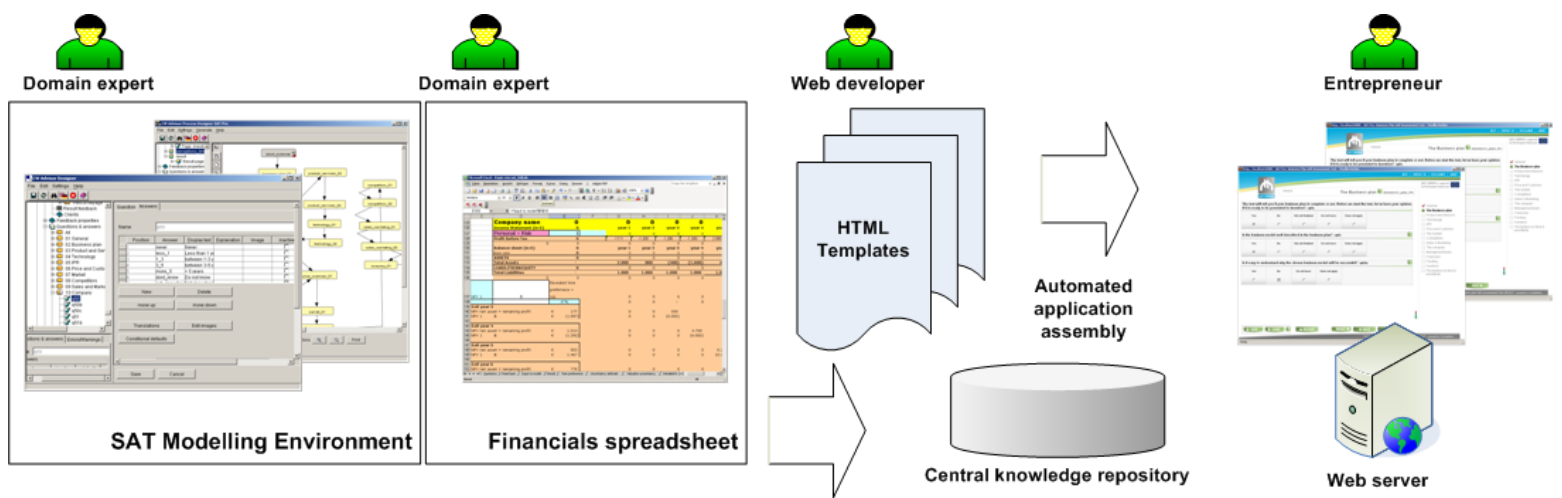

Figure 5: Architecture and development, stakeholders and development cycle.

assessment of the financial opportunities and risks of the investment, is derived. The typical figures on which investors' base their decisions with respect to financials are for instance Internal Return Rate, Price Earnings Ratio, or the Net Present Value of investments. The values need to be above a certain "hurdle rate" which depends on risk and maturity of the case in hand.

When such calculation schemes are to be made explicit and formalized in some software system, we observe that for many domain experts the preferred way of doing this is by means of a spreadsheet program. As the opportunities of this form of end user programming for specific tasks are well known (see for instance Nardi \& Miller's paper from 1990), we decided to adopt such a knowledge acquisition approach also in the SAT system. Thus, the domain expert can formulate and update the calculation schemes with a standard spreadsheet program. At run time, the SAT system directly interacts with this spreadsheet service: The values of the variables in $B P$ serve as inputs to the financial calculations and computed values are automatically transferred back to standard $B P$ variables, on which further expressions - like in feedback rules - can be defined. The only prerequisite for this tight integration of external spreadsheet logic is a defined mapping between $B P$ variables and spreadsheet cells.

\subsection{Overall architecture \& development cycle}

Figure 5 summarizes the overall architecture, stakeholder roles, and process flow of the SAT tool, see also (Felfernig et al. 2007) and (Jannach \& Kreutler, 2004) for technical details of the underlying ADVISOR SUITE framework. During design time, the domain expert - optionally together with a knowledge engineer - constructs or modifies the required business logic like the feedback rules or financial calculation schemes. All definitions except for the spreadsheet calculations are stored in a central knowledge repository. In parallel, a Web developer defines and maintains the HTML templates, which the framework uses for the generation of a corresponding Web application in an automated application assembly process. Once the resulting Web application is deployed on a Web server, it is ready for use for the entrepreneurs. The run-time components of the framework comprise among others a personalization agent which handles the interaction with the end user based on the definitions in the central knowledge repository, i.e., it for instance evaluates the current state of the interaction and determines the further flow of the dialog.

At the moment, the definitions in the knowledge base are static in a sense that they do not change automatically over time based, e.g., on the experiences gained from previous advisory sessions. The incorporation of such learning techniques is however part of our current and future work.

\section{DISCUSSION}

\subsection{Modelling and technology aspects}

For the SAT tool, a knowledge-based, integratedmodelling approach has been chosen due to the anticipated complexity of the business logic to be implemented. Currently, there are for instance more than 450 feedback fragments and accompanying selection rules stored in the knowledge base. The business plan profile comprises more than 120 possible direct questions, of which of course only a part is actually asked in one single dialog as all those questions are left out that are not relevant in the current context. The dialog model describing the different pages and the interaction flow (see Figure 4) is comparably small and contains about 40 
different interaction states. Note, that one such node can comprise several questions; in addition, individual questions are dynamically dimmed out based on personalization rules. Thus, the graphical dialog model remains at a manageable size. Compared to other ways of interaction modelling like dialog grammars (see, e.g., Bridge 2002), however, our state diagram approach is somewhat limited and static as all possible dialog paths have to be explicitly modelled. Still, from the perspective of modelling complexity, we claim that state diagrams are more comprehensible to users with limited IT background, given the declarative and implicit nature of grammar-based approaches. In future releases of the SAT modelling environment, however, we plan to incorporate the possibility of modelling sub-graphs in order to provide additional structuring mechanisms for complex graphs.

In general, the task of selecting appropriate feedback texts based on an interactively acquired user model can be accomplished by a conversational recommender system (see, e.g., Thompson et al., 2004) and a rule-based filtering approach. In contrast to previous work in this area, however, the SAT tool and the underlying ADVISOR SUITE system aim at providing not only algorithms for problem solving but also a comprehensive modelling environment which also includes domain-specific extensions like financial calculation schemes.

To the best of our knowledge, Ernst \& Young's IPO Navigator (see a report by Quittner, 1999) is the only other approach toward providing a comparable web-based electronic advisory service for entrepreneurs. While there exist some similarities between the IPO Navigator and the SAT tool from the end-user perspective, no reports on the internal implementation and the technological foundations of this tool are available.

Ernst \& Young's IPO Navigator is part of a larger set of electronic self-service tools developed within the company's broader e-service initiative. Comparably, the SAT modelling environment aims at providing a common technological basis for such types of applications as the internal mechanisms like, e.g., scoring or rule-based feedback, can be also applied to various other types of decision support and advisory problems. Up to now, two further related advisory applications not described in the paper (an entrepreneur personality check and another financials tool) have been built based on SAT technology.

\subsection{First practical results}

Currently, there are two versions of the SAT tool are in productive use. SAT LIGHT is a free, smaller version of the business plan advisory system which mainly covers the aspect of feedback rules and scoring. Furthermore, the questionnaire and the evaluation are not as detailed as in the full SAT PRO version, which also includes the detailed financial calculations. The SAT framework itself comprises a comprehensive logging component in which all user interactions are monitored and stored in the central repository. Thus, from the light version, which is online since spring 2005, first empirical results about the usage of the system are available. Up to now, about one thousand successful advisory sessions have been registered, in which an online user has fully stepped through the series of about 25 questions. From the overall usage statistics we see that from all users that go through the first five questions, about 50 percent make it to the final recommendation. We interpret this as a promising sign with respect to the end user acceptance and usability of the system, given the fact that online users in our opinion not easily accept such long "click-distances" on the Web, see also the study of (Smyth and Cotter, 2002) in the context of mobile portals. From our point of view, the more interesting type of information contained in the usage logs is the knowledge about common shortcomings of business plans or the typical characteristics of the entrepreneurs. On the importance of such aspects in the domain see for instance (Carswell \& Asoka Gunaratne, 2005). Although a variety of books and online guides exist about how to write effective business plans, to the best of our knowledge no statistical evidence is yet available about common mistakes and in particular about correlations along the different dimensions. Given such information, we hope that an in-depth analysis, which is part of our current research, can be a valuable contribution in particular in the area of entrepreneurship education.

At the moment, such an evaluation has not been done yet, mainly because the sample size of the more detailed SAT PRO tool is not yet sufficient. What can be reported from the SAT LIGHT tool yet are basic figures on individual numbers in the profile. So for instance more than a half of the online users had a combined practical management experience of less than four years, which indicates that most users of the tool are first-time entrepreneurs. Still, more than 70 percent are sure at the beginning of the analysis that their business plan is very clear about how the company will make money with its products and services. Even more, users also stated it would be easy to understand for investors why customers will pay for exactly their products, which indicates a common trend toward overestimation of the advantages and marketability of the new product. Another example would be the 
description of investor exit opportunities in the business plan, which is fully missing in 40 percent of the cases and which thus indicates that the "investor's view" is commonly not properly taken into account.

Compared with our own practical experience, the examples nicely reflect our observations of entrepreneurial attitude and investor perception during individual coaching sessions. The findings area also in line with the feed back received from more than 400 participants in the 30 Master Class seminars held throughout Europe during 2006 and 2007. Although it is too early yet to make wellfounded statements based on these statistics, the examples above should give a first impression about possible types of information contained in the interaction logs.

\section{CONCLUSION}

In this paper we have presented SAT, an interactive online advisory system in the area of technology and innovation commercialisation. The challenges with respect to the formalization of comprehensive domain expertise and the need for personalized user interactions have been discussed and we have shown how knowledge-based approaches can be exploited to minimize system development and maintenance efforts. In a broader view, we thus see our work also as a real-world case study of how such technologies can serve as an enabler of new e-business selfservice applications.

A first usage analysis indicate that such a system will be accepted by end users and as an alternative to classical means of information gathering and business plan evaluation like possibly expensive expert counselling. The "How to attract investors" Master Classes are all structured around the SATtool. More than 30 Master Classes have been held in 12 European countries. Practical experiences from contacts with more than 400 participants in these classes indicate that the tools also have a strong potential user base among innovation professionals. The tools thus provide an adequate structured approach to advising and coaching services offered to entrepreneurs by innovation professionals.

\section{ACKNOWLEDGEMENTS}

The work presented herein is partially supported by the Gate2Growth Initiative supported by the European Commission, D.G.Enterprise, see also www.gate2growth.com.

InvestorNet and the development of the SAT-tool have been supported by the European Commission, DG Enterprise.

\section{REFERENCES}

Bridge, D., 2002, Towards Conversational Recommender Systems: A Dialogue Grammar Approach, Workshop in Mixed-Initiative Case-Based Reasoning, Workshop Programme at the 6th European Conference in CaseBased Reasoning, pp. 9-22.

Carswell, P., Asoka Gunaratne, K., 2005, Exploring the role of entrepreneurial characteristics in determining the economic growth potential of an innovation, International Journal of Entrepreneurship and Innovation Management , 5(5/6), pp. $441-453$.

Felfernig, A. and Shchekotykhin, K., Debugging User Interface Descriptions of Knowledge-based Recommender Applications, in Paris, C. and Sidner, C. (Eds): Proceedings of ACM International Conference on Intelligent User Interfaces, Sydney, Australia, ACM New York, 234-241, 2006.

Felfernig, A., Friedrich, G., Jannach, D., Zanker, M., 2007, An Integrated Environment for the Development of Knowledge-Based Recommender Applications, Intl. Journal of Electronic Commerce, Special issue on Recommender Systems, 11(2), pp. 11-34.

Jannach D., Kreutler G., 2005, Personalized User Preference Elicitation for e-Services. In: Cheung W., Hsu J. (Eds.): The 2005 IEEE International conference on e-Technology, e-Commerce and e-Service, Hong Kong, IEEE Computer Society 2005, pp. 604-611.

Jannach D., Kreutler G., 2004, A Knowledge-Based Framework for the Rapid Development of Conversational Recommenders. Springer LNCS, 3306, pp. 390-402.

Kobsa, A., Koenemann, J., and Pohl, W., 2001, Personalized Hypermedia Presentation Techniques for Improving Online Customer Relationships, The Knowledge Engineering Review, 16 (2), pp. 11-155.

Nardi, B., Miller, R., 1990, The spreadsheet interface: A basis for end-user programming, Third Interational Conference on Human-Computer Interaction, pp. 977983.

Quittner, J., 1999, Testing Your IPO-Readiness on the Web, Business Week, November 25, 1999.

Smyth, B. and Cotter, P., 2002, Personalized adaptive navigation for mobile portals. Proceedings of the 15th European Conference on Artificial Intelligence, Lyon, France, pp. 608-612.

Thompson, C.A., Göker, M.H., and Langley, P., 2004, A Personalized System for Conversational Recommendations, Journal of Artificial Intelligence Research, 21, pp. 393-428.

van Noord, G., and Gerdemann, 2001, D., Finite State Transducers with Predicates and Identities. Grammars 4(3), pp. 263-286.

von Winterfeldt, D., and Edwards, W., Decision Analysis and Behavioral Research. Cambridge University Press, Cambridge, UK, 1986. 